Penitent Performance, Reconstructed Rumination or Induction: Student strategies for the deployment of reflection in an extended degree programme.

Vicki Trowler ${ }^{a *}$, Robert L. Allan ${ }^{a}$, Jarek Bryk ${ }^{b}$ and Rukhsana R. Din ${ }^{a}$ ${ }^{a}$ School of Applied Sciences, University of Huddersfield, Huddersfield, UK; ${ }^{b}$ Department of Biological Sciences, University of Huddersfield, Huddersfield, UK

*Vicki Trowler v.trowler@hud.ac.uk School of Applied Sciences, University of Huddersfield, Queensgate, Huddersfield HD1 3DH, United Kingdom 


\title{
Penitent Performance, Reconstructed Rumination or "Imagined Community" Induction: Student strategies for the deployment of reflection in an extended degree programme
}

\author{
While reflection has long been held to be central to learning, not all reflection leads to \\ insight or learning. Drawing on distinctions in psychology between "constructive self- \\ regulation" and "rumination" and educational literature which distinguishes \\ descriptive from reflective writing, this paper employs a novel approach to assign \\ student reflective writing to one of four quadrants (categorised as "selfie", "quick-fix", \\ "rumination" and "action") and uses this model to consider the reflection strategies \\ deployed by students in transition to student, disciplinary and professional identities. \\ Findings suggest that students who appear to master reflection adopt one of three \\ strategies: "reconstructed rumination", "induction" or "penitent performance". \\ Identifying which strategy a student is adopting can aid timely intervention to \\ facilitate student success.
}

Keywords: student reflection; reflective writing; student transition; rumination.

\section{Background}

Many claims are advanced for reflection within higher education. Reflection offers a bridge between experience and learning (Griggs et al. 2018) and offers the development of understanding in the learning process, rather than simply resolving problems (Pavlovich 2007). Students taught reflection, with sufficient scaffolding, can develop self-regulation and metacognitive skills, facilitating future learning (Mirriahi et al, 2018) and transformative learning (Carrington \& Selva 2010).

Reflection can be fostered through the use of technologies such as video or blogs (Mirriahi et al 2018), or through more traditional reflective journal writing (Pavlovich 2007; Wang et al 2017), with reflective skills improving through the academic year (Lew \& Schmidt 2011), provided that a varied and flexible approach is adopted (Griggs et al 2018), ethical issues are carefully navigated and trust upheld (Pavlovich 2007), clear guidelines established (Fisher 2003; Pavlovich 2007), and reflection embedded in lived experience (Smith \& Trede 2013). While some authors stress the importance of assigning credit for reflective writing to maximise student engagement with reflective assignments and to signal pedagogic valorising (Mirriahi et al. 2018; Pavlovich 2007), Wilson \& Howitt (2018) caution about the tension between creating a space for genuine reflection and a "surveilled" space encouraging performativity.

Grant et al. (2002:822) note that 
Purposeful progress through the cycle of self-regulation towards a specific goal rests on an individual's being able to monitor and evaluate his/her progress and use such feedback to improve his/her performance.

However, they draw a distinction between "constructive self-reflection" and "rumination", a form of contemplation they categorise as a dysfunctional state of self-absorption. Similarly, they distinguish between reflection and insight:

The processes of self-reflection and insight are logically independent. One may spend considerable time in self-reflection without gaining insight. (Grant et al. 2002:824)

They propose that self-reflection may be internally (self-) focused, or externally focused toward problem solving or solution seeking. Self-focused reflection, centred on seeking to "understand, contain or dissipate their negative emotional, cognitive and behavioural reactions", they argue (Grant et al. 2002:832) is less likely to lead to insight, changed behaviour and success in reaching goals. This process is illustrated in Figure 1, below. It is worth noting that Grant et al. (2002) found negative correlations between insight and depression, anxiety, and stress, but positive correlations between (forms of) self-reflection and measures of anxiety. This suggests that "rumination" may be linked to anxiety, and that anxiety, depression and stress may prevent (or arise from an inability to attain) insight within the cycle of self-regulation.

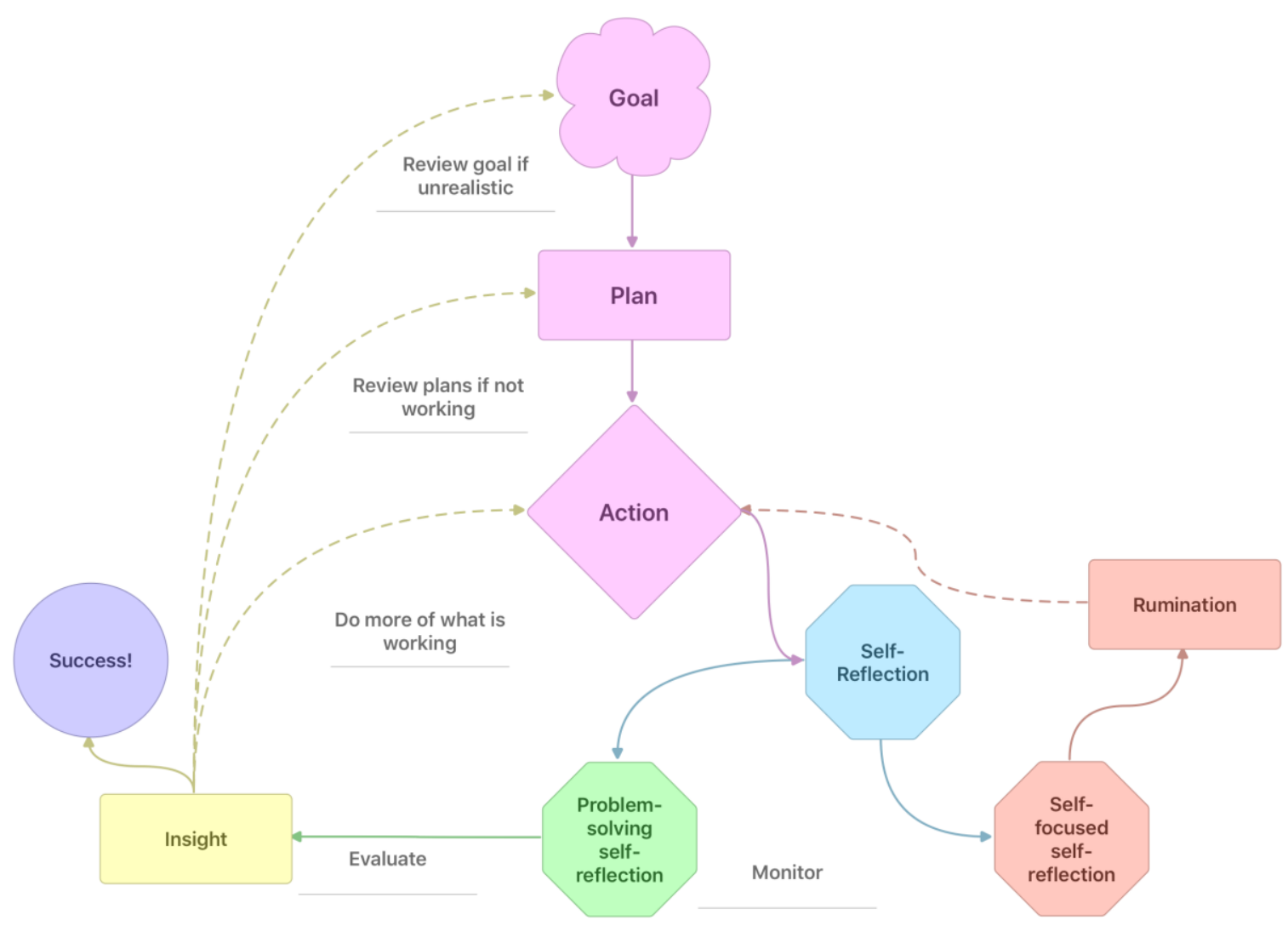


Figure 1: Self-regulation cycle (drawing on Grant et al. 2002)

Against this background, a university in the north of England offers an extended degree programme to students who do not qualify for direct admission to programmes in sciences. Many of these students have fallen short of obtaining the grades required for their desired programmes, while others find themselves attracted to careers or study programmes in science but have not studied science to the appropriate level at school / college. Still others are latecomers to higher education, having seen higher education as something for which they were never destined by virtue of their life circumstances.

These students typically present with at least one, but usually more than one, characteristic associated with poorer academic outcomes: many are first-generation students, often living at home with their families of origin and commuting to university; many hail from low-participation neighbourhoods, and less wealthy families, and often hold part-time or full-time jobs in addition to their studies; several are from black, Asian or minoritised ethnicity backgrounds; several have caring responsibilities, disabilities and / or other circumstances that constrain their ability to engage fully with, or prioritise, their studies.

For these students, the benefits offered by reflection (above) are even more valuable: for students whose performance or circumstances have left them feeling bruised or defeated, a chance to regain control, to remodel their academic orientation or to flourish in an environment in which they feel unqualified. Alongside disciplinary content, this extended programme offers crossdisciplinary skills, and uses regular reflective writing tasks alongside other modes of assessment.

Macfarlane and Gourlay (2009) caution against "emotional performativity" in reflective assignments, which they discuss in the context of Postgraduate Certificate in Education-type teaching portfolios. They caricature the process required in such programmes as "enacting the penitent self", akin to reality show contestants who follow a predictable path of penance, conversion and commitment. This leads, in Macfarlane and Gourlay's view, to conformity, inauthenticity and inequity, skewed against cultures, disciplines or dispositions that are less aligned to self-revelatory practices. While the focus of their paper was on learning and teaching programmes, the issues they raise may have currency in other HE contexts, especially if the reflection being elicited through reflective assignments becomes mired in self-focused rumination rather than problem-solving self-reflection and insight.

This paper examines the ways in which students on this extended degree programme in applied sciences deploy reflection during their studies. Identifying which strategy students are deploying can aid timely intervention to assist students in reaching their academic potential.

\section{Methods}

Owing to space constraints, details of methods followed - particularly on the quantitative side have been abridged. Fuller details including the scripts used to classify the data and to generate the plots, and full references for software used, are available on a purpose-built website, at: 
https://veryinterestingresearchmethods.weebly.com. ${ }^{1}$

As part of their course requirements, students on the programme were required to submit regular reflective blog posts on topics related to their motivations, study behaviours, intended destinations after completion of the programme and experiences of the programme. An example topic asked "Has your understanding of how you as an individual learn changed since the start of the course and if so in what way? Which, if any, aspect/s of your educational development do you still need to work on, and do you feel you are on track to pass the course with the grades you would like?" While these submissions were graded according to the academic requirements of the set assignments, they were also later analysed as data for this study. Ethical approval was obtained through the appropriate School channels, and all research procedures conducted consistently with guidelines and good practice. Students voluntarily provided informed consent.

Mixed methods were employed in this study. These components proceeded in parallel with each other, enriching all processes. Text analysis methods were harnessed to sort the blog posts into categories, as described below, while a traditional qualitative analysis of the posts drew out themes and provided "thick description" to understand what was happening within each of the categories.

\section{Classifying the data}

Literature on reflective writing, and particularly on assessing the reflective writing in student assignments, typically differentiates between levels of reflection, with the most basic levels typically deemed purely descriptive, and higher levels acquiring more sophistication and criticality. Because the length of typical reflective assignments in the study programme was only a couple of hundred words, it was recognised that this did not provide students with the opportunity to display greater levels of sophistication and criticality in their reflection.

Because of this, it was decided not to discriminate finely on depth of reflection, but to deploy instead a simple binary (primarily descriptive vs reflective) for the purposes of this analysis. Similarly, writing was deemed either primarily self-focused or problem-solving in its reflective orientation, giving rise to a $2 \times 2$ matrix. The quadrant names for the "primarily reflective" posts were derived from the two types of reflection proposed by Grant et al. (2002). The names for the "primarily descriptive" quadrants were coined to differentiate these from the other quadrants: "quick fix" to denote a focus on problem-solving, but with less reflection than the "action" quadrant; and "selfie" to denote a focus on the self, but without the level of reflection denoted by "rumination". There is no intention to connote vanity or narcissistic self-absorption - rather, we recognise the "selfie" as being a valid means by which young people "perform the self" (see Jackson 2019). This classification schema is shown in Table 1.

\begin{tabular}{|l|l|l|}
\hline & Primarily Self-Focused & Primarily Problem-Solving Focus \\
\hline Primarily Reflective & Rumination & Action \\
\hline
\end{tabular}

\footnotetext{
${ }^{1}$ After anonymous review, more orthodox means of providing access e.g. posting code on GitHub will be followed.
} 


\begin{tabular}{|l|l|l|}
\hline Primarily Descriptive & "Selfie" & "Quick-Fix" \\
\hline
\end{tabular}

Table 1: Classification Schema for Reflective Assignments

Each blog or video by each student was assigned to a quadrant on the following basis: The depth of reflection (primarily descriptive vs reflective) was derived from the feedback allocated to the assignment submission by the original marker. Assessing depth of reflection has been the subject of much debate in the literature (see Fisher 2003; Pavlovich 2007), with issues of inter-coder agreement generating concerns regarding validity and reliability (see Lew \& Schmidt 2011). The use of computers to assess depth of reflection is the subject of ongoing study (see, e.g. Ullman 2019); however, while authors such as Lew \& Schmidt (2011) employ computerised content analysis to determine whether writing demonstrates reflection or not, we find little evidence in their methods including the generation over three months of a custom-built, but unvalidated, lexicon - to indicate that reflection can conclusively be determined. (The presence of terms such as "reflection" in student writing does not indicate, in our view, that reflection has taken place.) For this reason, we have for the moment relied on human assessment of the depth of reflection.

With regard to the focus of reflection, the blog posts and transcriptions of the videos were scored by computer using the Regressive Imagery Dictionary. This well-validated lexicon produces a score of "primordial thinking" (as opposed to "conceptual thinking") for a piece of text (Martindale, 1976; Martindale, 1990). In this context, "primordial thinking" with its more inward direction served as a proxy for "self-focused" reflection, while "conceptual thinking" with its more outward focus served as a proxy for "problem-solving" focused reflection".

For the purposes of this study, the procedure was amended to produce a score of "conceptual thinking", with results greater than zero indicating a preponderance of "problemsolving" focused reflection and results less than zero indicating a preponderance of "self-focused" reflection. To minimise the effects of bias introduced by the topic itself, each topic was also scored using the Regressive Imagery Dictionary, and the topic score subtracted from each student's post score for that topic.

Figure 2 displays the results of mapping a single point for the mean value of all blog posts, for each student, of the 2017-18 cohort. The letter assigned to each point, in Figure 2, shows the mean of each student's final grades, with A being 70\% and above, B $60-69 \%$, C $50-59 \%$, D $40-$ $49 \%, \mathrm{~F} 39 \%$ and below, and other symbols representing students who withdrew from or did not complete the programme.

2 "Conceptual thought is abstract, logical, reality oriented, and aimed at problem solving. Primordial thought is associative, concrete, and takes little account of reality." https://provalisresearch.com/products/content-analysis-software/wordstatdictionary/regressive-imagery-dictionary/ 


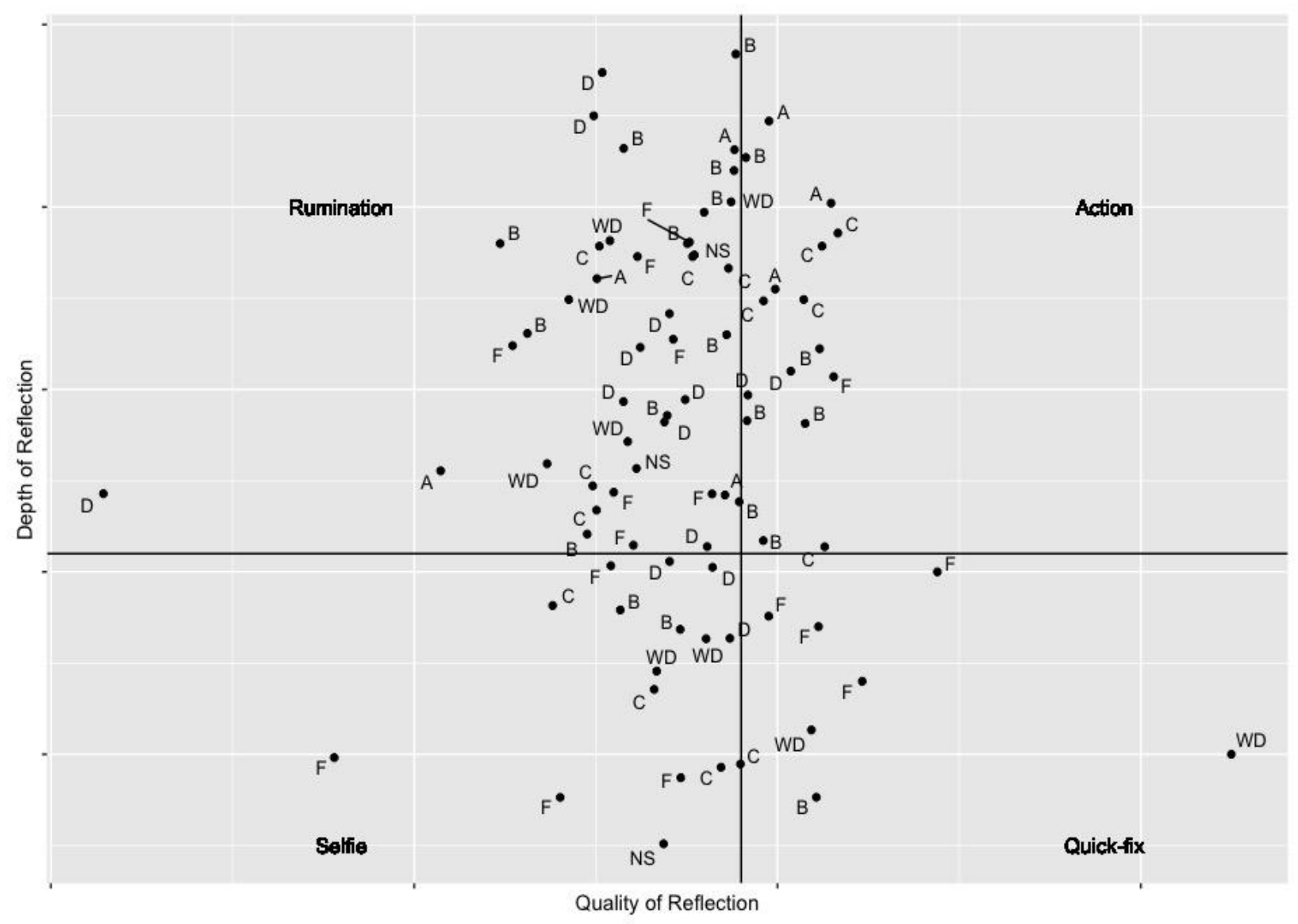

Figure 2: Mean value of Depth of Reflection vs Focus of Reflection, for all students in a single cohort showing mean final grade

\section{Qualitative analysis}

Data were analysed using NVivo, following a process similar to that described by Charmaz (2014:109-191) with initial coding of the data involving line-by-line reading and highlighting noteworthy phrases. Working through the data, similarities and differences between and within blogs and videos were sought and more meaningful names were assigned to the initial codes. This initial coding was progressively augmented with focused coding, organising the codes into categories and teasing out relationships between these categories. This process was revisited a number of times, tweaking and adding codes as newer data pushed themes to the fore or backgrounded other codes. 


\section{Findings}

As seen in Figure 2, students appear at different points on the graph. This is unsurprising owing to the diversity of students on the programme. Many of the best academic performers (students labelled A and B in Figure 2) in the "action" quadrant, and many of the lowest performing students (labelled F) in the "selfie" and "quick-fix" quadrants: if the model holds, this is consistent with expectations. (Statistical tests are available at https://veryinterestingresearchmethods.weebly.com )

Amongst the highest scores on the $y$-axis (measuring "depth of reflection") are some students with lower mean academic grades ( $D$ or F). While the capacity for deeper reflection ought to translate into stronger academic performance, according to the literature on reflection cited earlier, this apparent lack of correlation between "depth of reflection" and academic performance suggests that the "quality of reflection" is at least as important: that is, that it is not just how well students are reflecting, but the nature of that reflection ("self-focused" vs "problem-solving focused") that matters. This would appear consistent with Grant et al. (2002)'s hypothesis. These students may be considered to be performing "reconstructed rumination" - their posts suggest that they have mastered the capacity of reflection, but their reflection has been predominantly "selffocused", and has not translated into insight, nor produced stronger academic performance.

However, a number of non-intuitive observations warrant further consideration. These include high-performing students (labelled A or B) whose posts fall predominantly in the "quick-fix" and "selfie" quadrants or on the left-hand side of the "rumination" quadrant, as well as lowerperforming students (labelled F) whose posts fall predominantly in the "action" quadrant and toward the right-hand side of the "rumination" quadrant. To consider what may be happening here, we need to move from quantitative representations to qualitative examinations of the students' blog posts. We begin by considering each quadrant through illustrative examples of posts from that quadrant, before going on to consider the "less intuitive" observations.

\section{Selfie Quadrant:}

The "selfie" quadrant is defined by lower scores on depth of reflection (i.e. the writing is more descriptive than reflective) and a greater focus on "self" than on "problem-solving", in the quality of reflection. This may manifest in the following ways, as exemplified by quotes from student blog posts:

The inward focus reflected in posts in this quadrant remains at a descriptive level, with little evidence of reflective engagement:

I am making small changes in order to make my engagement rate higher and effect...

my personal self in a positive way. I have changed because... this has really helped with my individual self as I needed to realise myself.

\section{- $\quad$ Student 3}


Student posts from this quadrant may also demonstrate an externalising of the locus of control. This construct (Rotter 1966) reflects the extent to which someone perceives themselves to have agency over a situation: someone exhibiting an internal locus of control in a situation would consider themselves to author the outcomes through their behaviours, while someone exhibiting an external locus of control may ascribe the outcomes as due to luck, to others, or to factors over which they have little or no control. (Given the demographics and life histories of many of these students, it is not unreasonable for many of them to experience agency as highly constrained. This should not be read as judgmental, but as diagnostic in terms of signalling where support may be needed.) An external locus of control has been positively correlated with lower socio-economic status, lower achievement, ethnicity, and younger age in children (see Nowicki \& Strickland 1973). Bar-Tal and Bar-Zohar (1977:183) argue that an external locus of control leads to "little reason to exert taskoriented effort in an attempt to improve... academic achievement". This can also manifest retrospectively, with disappointing performance being ascribed to external factors rather than the behaviour of the student:

...there are various reasons why I did not achieve my grades some where [sic]... down to staffing issues and me not getting the right support to help reach my full potential on my course.

\section{- $\quad$ Student 1}

External locus of control is also negatively correlated with independent learning (Nowicki \& Strickland 1973) in higher education (particularly male) students. While also negatively correlated with motivation, this struggle to gain independence in learning may arise from the student's perceived lack of agency over their learning. For a number of students, this centred around a discourse of "asking for help", rather than a more agentic enlisting of resources:

University is a step up from college... University is much more independent and it has meant that I have had to take more time to understand the material by myself... Learning from lectures is a new thing for me as in college, we had more structured lessons with more help and support. I must be an active listener as well as make my own notes from scratch. There is help and support but it is not as accessible as it would be at college so I have to be more assertive.

\section{- $\quad$ Student 14}

This discourse of "asking for help" on occasion manifests in the construction of independence as problematic:

The lessons that I have learned from [underperforming at school] was I did not bother asking my teachers for help when I was struggling with my coursework, but instead I keep it to myself thinking that I can do it. This year, I will change my attitude and 
be mature student. I will make sure to ask help from my teachers whenever I feel like I need it...

\section{- $\quad$ Student 2}

Student posts from within this quadrant thus suggest students struggling with having agency over their learning. With many students on the programme having backgrounds which include factors which are positively correlated with externalising the locus of control, this should not be surprising. The positive correlation of external locus of control with lower performance merits attention, particularly as negative correlations have also been found between external locus of control and cognitive approaches, such as the seeking, sorting, and application of useful information in the execution of tasks (Bar-Tal \& Bar-Zohar, 1977).

\section{Quick Fix Quadrant}

The "quick-fix" quadrant is defined by lower scores on depth of reflection (i.e. the writing is more descriptive than reflective) and a greater focus on "problem-solving" than on "self", in the quality of reflection. This manifests in the following ways, as exemplified by quotes from student blog posts:

While externally focused, a number of blog posts in this quadrant demonstrate a tendency toward universalising, projecting the issue into an abstract, immutable law instead of concretising the issue in the context of their own experience and taking ownership of the lessons learned. This is illustrated by the following examples:

there are many factors contributed to get advance level of knowledge for example the time of studying the revision technique and the idea of note taking.l know that no one can cover everything in the blink of an eyes and every achievement needs an effort and no success without work but to have all factors do not mean you will success unless if you organised yourself and and create your own setting-goals. [sic]

\section{- $\quad$ Student 4}

Time is precious and should not be wasted. It is important to revise daily... It is essential to understand the topics in the lectures because when it comes to revising it later on for the exam it will still be in your memory. Whereas if you don't go over what you learnt in your lectures then you will easily forget and will struggle when it comes to revising so you should never leave revision to the last minute, especially if you haven 't gone over beforehand.

\section{- $\quad$ Student 5}

These posts show a generic, distanced engagement with the subject matter, in stark contrast to the 
more reflective posts found in the "action" quadrant. Another tactic employed in posts in this quadrant demonstrates taking a superficial or instrumental approach to learning. The first example illustrates a student recognising that in order to gain "the required marks to pass", a small adjustment in behaviour was required, leading to a rather superficial level of learning:

At first I thought it would be 'a walk through the park' as I have completed many [similar] tests in the past. However I learnt that I had to know a lot more than I thought as this was a lot more in [depth]. At my first attempt I did not get the required marks to pass this was mainly due to not reading the questions properly which had many consequences. Now I know I should read the questions properly and also revise properly and not to rush as I realised this is what I did which had a bad effect on me.

\section{- $\quad$ Student 6}

The following example demonstrates an instrumental approach to learning, where the student refuses to consider the possible value of engaging with the assignment, since their current strategy appears to be delivering the required results:

I honestly have absolutely no idea how many hours I have worked per week, it is something I personally don't see being relative and monitored. When it comes down to my engagement throughout the week, I look at it from a different perspective, I have handed in all outstanding work on time... and in the summative assessments I have passed them all so far. So to summarise I believe that I am doing enough work in and out of university to be able to pass all the important assessments so far this year.

- $\quad$ Student 7

While the former two examples illustrate disengagement through distancing, the latter two illustrate disengagement through a more strategic approach. This involves either conceding to small adaptations to behaviour in order to meet the minimum requirements, or refusing to engage since the minimum requirements are already being met.

\section{Rumination Quadrant}

The "rumination" quadrant is defined by higher scores on depth of reflection (i.e. the writing is more reflective than descriptive) and a greater focus on "self" than on "problem-solving", in the quality of reflection. This manifests in the following ways, as exemplified by quotes from student blog posts:

In contrast to the posts in the "quick-fix" quadrant, posts in this quadrant demonstrate a readiness to engage with reflection and self-critique. The first example illustrates a willingness to recognise shortcomings, even when the minimum requirements are being met: 
The aspects of educational development that I feel I still need to improve on is time management and independent study, this has always been an issue I find it very difficult to sit down and do independent work or assignments, I put it off for so long that it gets to the point in most cases where I need to rush it to get it done, not so bad that I need to stay up late but to the point where I'm maybe not putting the most amount of effort or quality into it.

\section{- $\quad$ Student 8}

The second example deploys textual markers to signal a ruthless self-appraisal, such as "I can honestly say, this isn't sufficient", or "I feel as though I have kept myself busy...":

However, in terms of private study I have spent very minimal time for example; spent minimal hours on revision, covering notes, extra reading, preparing before a lecture etc. and I can honestly say, this isn't sufficient enough... I feel as though I have kept myself busy with other commitments such as deadlines ... and therefore spent less time on revision so although I spend a good amount of hours on my studies I need to focus on the right areas of study such as those modules which I find more difficult than others..

- $\quad$ Student 9

In the third example, the student acknowledges external factors as well as their own response to those as contributing to their sub-par engagement, and signals an acknowledgement of the consequences of this:

Over the course of this term, I think my engagement in this course has been very poor... I think this is because I've had a difficult time adjusting in to university life as I moved away from home and into university halls. I think that I have not been taking my tests and assignments seriously enough as many of them are formative. However, I know [sic] realise that formative tests are a good indicator of where you are in each subject and they can be helpful for my improvement. Moreover, because of my lack of engagement during this term, it is hard for me to see whether I am actually finding the modules difficult as I have not given my best effort. Although my engagement at home has been very poor, I have managed to keep my attendance high as I engage more in lectures than at home

- $\quad$ Student 10 
In the example below, the student concedes that while there are external factors, ultimately their inadequate engagement is down to their own choices, which they describe as being poor:

I live in student halls so there are often a lot of distractions as my flatmates are all studying different courses so we have different deadlines so sometimes when I have a deadline to meet during a certain week, my flatmates may not have much work to do so they may make arrangements to watch movies and I often join them even if I know that I have a deadline... realistically I have enough time to spend 35 hours a week on study. However, I feel a lot of pressure socially which often results in me staying up late at night socialising instead of going over my lecture notes and instead of doing my assignments on time. I feel as if, I have not been managing the competing demands well at all. This has resulted in me feeling as if I am not only unsatisfying myself but also everyone else.

\section{- $\quad$ Student 10}

In these posts, students feel that they have a good awareness of what their problems, issues and constraints are - but they feel "stuck", not empowered to make the changes they recognise as necessary. Unlike posts in the "selfie" quadrant, these posts show students taking responsibility for their shortcomings; however, they demonstrate the performance of reflective self-flagellation rather than showing students acting on the realisation of the shortcomings to change their behaviour.

\section{Action Quadrant}

The "action" quadrant is defined by higher scores on depth of reflection (i.e. the writing is more reflective than descriptive) and a greater focus on "problem-solving" than on "self", in the quality of reflection. This manifests in the following ways, as exemplified by quotes from student blog posts:

The posts in this quadrant show students taking responsibility for their learning through reflecting on their shortcomings, and putting plans into action to address these. In the first example, the student considers changes they have made to their approach to learning, reflecting on the distance travelled and the distance still to cover:

I have reflected on [my previous approach to learning] and researched that it is a lot more beneficial when one views lecture material before the lecture, to familiarise and gain the most benefit of pedagogy environment. By doing this, the time spent in the lecture is much more efficient and rewarding.... This has allowed me to develop my learning significantly since the beginning of the course. However, it is my ability to revisit and review the material which I feel is lacked in terms of my educational development. I have found that revisiting material allows one to retain information from short term memory into long term memory and is significant in educational 
development. This is a skill, which has already been put into practice and I hope to perfect in the near future.

\section{- $\quad$ Student 11}

Similarly, the example below engages with what the student has done to change their approach, what their experience of this has been, where they still feel the need to improve, and what they intend to do to address this:

Previous to this course my understanding of learning was very little I believed if I memorise all the fact and textbooks I could easily pass. It is what I was taught in high school all the way to sixth from by teacher drumming into me just memorise and complete past exam questions. However, since starting this course my understanding has changed drastically and is still changing ...Since starting this course I have learnt learning isn't instant and I shouldn't be hard on myself if I'm not understanding straight because nobody does... I have found that when I put the effort and patience into physics and I am slowly and steadily beginning to understand. I have used different revision techniques to improve. I no longer count memorising exam techniques as helpful at all ...I believe I still need to work on my higher order thinking and learning skills such as evaluating and analysing however, I understand that I won't understand or get these skills correct or gain the marks I thought straight [away]... However, by practicing these skills through my labs and... essay I hope I can improve these skills for future assignments.

\section{- $\quad$ Student 12}

In the third example, the student recognises resources that they have at their disposal that they have not yet drawn on sufficiently, in order to address outstanding issues, and draws on that to feel empowered to improve:

At the Beginning of the course I didn't feel I had any direction in my learning, it felt more like I was blundering around just hoping for the best... Over the past three months I have improved my note making skills drastically, and no longer find myself re-writing my notes to make them all neat and pretty; instead I add extra notes to previous ones and highlight different areas to make the information stand out if important. I still really need to work on my time management skills, though I have improved majorly since September, I still need to utilise the resources around me more such as my wall 
planner or and excel document to make a weekly timetable... [I] know what I need to do now to improve and that is oddly calming...

\section{- $\quad$ Student 13}

Unlike the posts in the "rumination" quadrant, posts in the "action" quadrant demonstrate a sense of agency and empowerment, with students acting on their reflection to take responsibility not just for their shortcomings but also for their continued improvement in their learning.

\section{Non-intuitive observations}

Lower-performing (in terms of final academic grades) students whose mean scores across all of their blog posts position these in the "action" quadrant appear to be anomalous. There are several possible explanations, including:

- these students may be reflecting to the standard, and in the manner, desired, but have been unable to perform strongly in the module assessments. This is less likely, since the six modules represent a variety of assessment modes. Where extenuating circumstances have been reported, this has been reflected in the symbols used, e.g. EC rather than F;

- these students may be reflecting to the standard, and in the manner, desired, but are unable to transfer this capacity to effect better academic outcomes. Like students who score highly in second or subsequent language studies, but are unable to function in such languages, it may be that students have approached these tasks in a purely cognitive way, rather than more holistically. However, this can be argued to be a subset of the explanation immediately below;

- these students have worked out what is required, but are producing this mimetically and inauthentically; in Macfarlane \& Gourlay's terms, they are enacting "penitent performance". They are driven more by the need to comply than the desire to become, and draw their motivation from external sources.

The other group that warrants particular consideration are the group of higher-performing (" $\mathrm{B}$ average") students whose posts are predominantly found in the "selfie" and "quick fix" quadrants. These three students, it might be thought, resist reflection and may not be making full use of the opportunities afforded them on the programme. Possible explanations might include:

- these are students with personal or cultural barriers to reflection, who are nonetheless able to learn in a robust way. This may have been a factor in the case of one student, further compounded by language barriers;

- these students are "strategic learners", and are choosing to invest their time and effort into "higher yield" activities which generate more marks - such as exams, longer essays or lab reports - rather than reflective blogs. This does appear to have played some role with at least one student;

- these students have had prior exposure to the work covered in the academic modules, and are relying on that rather than gaining new skills, knowledge and insight. This appears to have been a factor with at least one student; 
- students may have shown stronger performance across modules where reflection was not required, and weaker performance on modules where reflection was more significant. Closer examination of students' results shows this was significant in all cases.

\section{Discussion}

In considering the anomalies found in the "Action" quadrant, we found the work of Stenhouse useful. Stenhouse (1975) identifies four component processes of education, namely training, instruction, initiation and induction. Where training is considered the acquisition of skills, and instruction the learning of information, initiation is postulated as the gaining of familiarity with social values and norms, and induction as the suffusion of the culture and thought system. Thus, while an initiate may perform congruently in a predictable manner, an inductee's behaviour, while highly congruent, is far less predictable, since $\mathrm{s} /$ he has internalised the culture and is deriving motivation from this internal locus rather than the external locus of the initiate, whose behaviour is more mimetic. Ashwin \& Komljenovic (2018:127) argue that HE becomes transformative when

...students' sense of self is changed through their engagement with university and with knowledge. This involves students relating their personal projects to the world and seeing themselves implicated in knowledge.

This would require induction, rather than initiation, instruction or training. Similarly, "problemsolving" focused reflection ought to indicate ownership of knowledge (Dyment \& O'Connell 2011) or students "examin[ing] their beliefs, values, experiences and assumptions about the subject matter at hand" (Minott 2008) - congruent with Ashwin \& Komljenovic's (2018) "transformative HE". "Problem-solving", outwardly focused reflection is thus likely to occur in contexts where education involves induction, and to allow for HE to fulfil its transformative potential.

We are thus proposing that the "Action" quadrant in fact contains two different types of behaviour: "penitent performance", and "induction". Unlike "penitent performance", where behaviour is mimetic, induction requires internalisation of the culture and thought systems of the university, discipline and / or profession with which inductees identify, and reflection from an internal, though outwardly focused, locus.

By contrast, the focus in "reconstructed rumination" is inward. While initiates may have become familiar with norms and values through "initiation", they have not yet reached the confidence of inductees to adopt behaviours that are both congruent and authentic. Unlike "penitent performance", which involves the ability to enact congruent behaviours inauthentically, "reconstructed rumination" invokes a sense of being trapped between the desire to adopt congruent behaviours and the need to behave authentically - recognising that behaviours these students observe as congruent do not feel authentic to them.

A useful analogy might involve instructing a group of people to draw a live model, posing in front of them. The "selfie" group might produce a stick figure; the "quick-fix" group might take a photograph and apply a "drawing" filter. "Reconstructed ruminators" will attempt the drawing, constantly erasing and making minor adjustments, hampered by their "inability to draw", ultimately not producing a complete drawing. The "penitent performers" might photograph the model, but unlike the "quick-fix" group, they will recognise that applying a filter is inadequate, and will use a 
drawing app to trace over the photograph - producing a hand-drawn artefact, albeit a traced one. The "inductees", by contrast, will recognise that the exercise involves looking at and interpreting the model and transferring what one sees onto paper, rather than aiming for a perfect reproduction, and will make a drawing of whatever level of representative reality. And while it may be difficult to tell some of the drawings produced by "penitent performers" apart from some drawings produced by "inductees", the process by which those drawings have been created differs, and thus too the nature of the learning.

Given the variety of backgrounds, experiences and orientations of students in the programme, it is not surprising that students deploy a range of reflection strategies. Identifying which strategy a student is adopting can aid timely intervention to assist students in reaching their academic potential. (This is considered in more detail in a forthcoming paper by the authors.)

\section{Conclusion}

While the importance of reflection in learning has long been accepted, not all reflection leads to insight or improvement. Grant et al. (2002) propose that reflection may be "self-focused" or "problem-solving" focused, leading to either rumination or action. Combining this proposal with insights from the higher education literature on reflection, this paper has proposed a four-quadrant model for classifying student reflective writing, and has used this to consider the reflection strategies deployed by students.

While posts can relatively straightforwardly be assigned to the four quadrants according to the depth and nature of reflection, some "non-intuitive" placements suggest that students who appear to master reflection adopt three distinct strategies: "reconstructed rumination" involves becoming adept at reflection, but without translating that into insight or action, posting mainly in the "rumination" quadrant; "induction" requires the ability to harness reflection and use this congruently with the culture and thought systems of their discipline to learn transformatively; while "penitent performance" suggests the observation of the social values and norms and the mimetic ability to produce reflective writing of the required standard and type, without internalising the learning.

Identifying which strategy a student is adopting can aid timely intervention to facilitate student success, since students deploying "penitent performance" may appear to be adept at "problem-solving" reflection but may not be reaping the benefits of reflection or transferring these to their learning.

\section{References}

Ashwin, P., \& Komljenovic, J. (2018). The conceptualisation of students' personal transformation through their engagement in South African undergraduate education. In P. Ashwin, \& J. M. Case (Eds.), Higher education pathways: South African undergraduate education and the public good (pp. 125-35). Cape Town: African Minds. 
Bar-Tal, D., \& Bar-Zohar, Y. (1977). The relationship between perception of locus of control and academic achievement. Contemporary Educational Psychology, 2(2), 181-199. doi:10.1016/0361-476X(77)90020-0

Carrington, S., \& Selva, G. (2010). Critical social theory and transformative learning: Evidence in preservice teachers' service-learning reflection logs. Higher Education Research \& Development, 29(1), 45-57. doi:10.1080/07294360903421384

Charmaz, K. (2014). Constructing grounded theory (2nd ed.). London: Sage.

Dyment, J. E., \& O'Connell, T. S. (2011). Assessing the quality of reflection in student journals: A review of the research. Teaching in Higher Education, 16(1), 81-97. doi:10.1080/13562517.2010.507308

Fisher, K. (2003). Demystifying critical reflection: Defining criteria for assessment. Higher Education Research \& Development, 22(3), 313-325. doi:10.1080/0729436032000145167

Grant, A. M., Franklin, J., \& Langford, P. (2002). The self-reflection and insight scale: A new measure of private self-consciousness. Social Behavior and Personality: An International Journal, 30(8), 821-835. doi:10.2224/sbp.2002.30.8.821

Griggs, V., Holden, R., Lawless, A., \& Rae, J. (2018). From reflective learning to reflective practice assessing transfer. Studies in Higher Education, 43(7), 1172-1183. doi:10.1080/03075079.2016.1232382

Jackson, H. (2019). Self[ie] reflective practice: Revealing student engagement through the photographic performance of the self. Learning, Media and Technology, 44(2), 144-161. doi:10.1080/17439884.2018.1563107

Lew, D. N. M., \& Schmidt, H. G. (2011). Writing to learn: Can reflection journals be used to promote self-reflection and learning? Higher Education Research \& Development, 30(4), 519-532. doi:10.1080/07294360.2010.512627

Macfarlane, B., \& Gourlay, L. (2009). The reflection game: Enacting the penitent self. Teaching in Higher Education, 14(4), 455-459. doi:10.1080/13562510903050244

Martindale, C. (1975). Romantic progression: The psychology of literary history. Washington DC, USA: Hemisphere.

Martindale, C. (1990). The clockwork muse: The predictability of artistic change. New York, USA: Basic Books.

Minott, M. A. (2008). Valli's typology of reflection and the analysis of pre-service teachers' reflective journals. Australian Journal of Teacher Education, 33(5), 55-65. 
Mirriahi, N., Joksimović, S., Gašević, D., \& Dawson, S. (2018). Effects of instructional conditions and experience on student reflection: A video annotation study. Higher Education Research \& Development, 37(6), 1245-1259. doi:10.1080/07294360.2018.1473845

Moon, J. (2001). Reflection in higher education. York: The Higher Education Academy.

Nowicki, S., \& Strickland, B. R. (1973). A locus of control scale for children. Journal of Consulting and Clinical Psychology, 40(1), 148-154.

Pavlovich, K. (2007). The development of reflective practice through student journals. Higher Education Research \& Development, 26(3), 281-295. doi:10.1080/07294360701494302

Rotter, J. B. (1966). Generalized expectancies for internal versus external control of reinforcement. Psychological Monographs, 80(1), 1-609.

Smith, M., \& Trede, F. (2013). Reflective practice in the transition phase from university student to novice graduate: Implications for teaching reflective practice. Higher Education Research and Development, 32(4), 632-645. doi:10.1080/07294360.2012.709226

Stenhouse, L. (1975) An introduction to curriculum research and development. London: Heinemann.

Ullmann, T. D. (2019). Automated analysis of reflection in writing: Validating machine learning approaches. International Journal of Artificial Intelligence in Education, doi:10.1007/s40593019-00174-2

Wang, H., Chen, H., Lin, H., \& Hong, Z. (2017). The effects of college students' positive thinking, learning motivation and self-regulation through a self-reflection intervention in Taiwan. Higher Education Research \& Development, 36(1), 201-216. doi:10.1080/07294360.2016.1176999

Wilson, A. N., \& Howitt, S. M. (2018). Developing critical being in an undergraduate science course. Studies in Higher Education, 43(7), 1160-1171. doi:10.1080/03075079.2016.1232381 